\title{
A Survey on Wandering Behavior Management Systems for Individuals with Dementia
}

\author{
Arshia Zernab Hassan ${ }^{1}$ \\ Computer Science Department \\ University of Minnesota \\ Duluth, Minnesota, USA
}

\author{
Arshia Khan ${ }^{2}$ \\ Computer Science Department \\ University of Minnesota \\ Duluth, Minnesota, USA
}

\begin{abstract}
Alzheimer's and related dementia are associated with a gradual decline in cognitive abilities of an individual, impairing independent living abilities. Wandering, a purposeless disoriented locomotion tendency or behavior of dementia patients, requires constant caregiver supervision to reduce the risk of physical harm to patients. Integrating technology into care ecology has the potential to alleviate stress and expense. An automatic wandering detection system integrated with an intervention module may provide warnings and assistive suggestions in times of abnormal behavior. In this study, we survey existing research on technology aided methodologies and algorithms used in detection and management of wandering behavior of individuals affected with dementia. Our study provides insights into mechanisms of collecting movement data and finding patterns that distinguish wandering from normal behavior.
\end{abstract}

Keywords-Dementia; wandering behavior; technology; algorithm;

\section{INTRODUCTION}

Dementia is a neuro-degenerative disease that decreases independence. Dementia affects the lives of $\sim 47$ million people worldwide [1], which is estimated to increase to 131.5 million in 2050. According to the Alzheimer's report from 2016, around 5.5 million Americans suffer from Alzheimer's dementia resulting in medical expense (professional caregiver and treatment cost) of $\$ 259$ billion. Family members (unpaid, unprofessional caregivers) spend 18.2 billion hours per year amounting to $\$ 230.1$ billion [2].

Dementia is sometimes revealed through 'wandering', which is a pervasive behavioral symptom in dementia patients [3]. It is defined as "a syndrome of dementia-related locomotion behavior having a frequent, repetitive, temporally disordered, and/or spatially-disoriented nature that is manifested in lapping, random, and/or pacing patterns, some of which are associated with eloping, eloping attempts, or getting lost unless accompanied" [4]. It may be triggered by various factors such as frustration, the intent for socialization or work, boredom or escaping tendencies [3]; however, it is quite unforeseeable and therefore requires supervision for detection and arbitration. Unattended aimless roaming of a patient may lead to agitation, fatigue, vertigo and in extreme cases physical harm due to falling or colliding with objects in the vicinity [2]. Moreover, wandering has been identified as one of the main reasons for nursing home placement or institutionalization [5], as it has proven to be too arduous for caregivers to manage in home environments.
Technological intervention, for detection and mediation of wandering behavior, would share the load of human labor and may also improve the privacy and independence of the patient. For example, an automatic wandering detection module can be integrated with an intervention module (i.e. for generating alert signals) to build a real-time system to produce prompt warnings [6]. This would help in reducing immediate health hazards associated with the aimless movement. Additionally, wandering behavior is correlated with the cognitive state of a dementia patient. Automatically generated records of wandering frequency and patterns would aid in keeping track of patients' cognitive health. As mentioned before, wandering behavior requires a considerable amount of caregiver vigilance; an automated technological solution has the potential to lower caregiver burden as well as medical cost.

A comprehensive survey or review on technological interventions for wandering management would contribute to research efforts in computation and cognitive health sectors and create a platform for future studies. As a precursor to formulating a robust algorithm for detecting wandering behavior, a survey on existing systems, would help delineate practical and effective approaches along with limitations, disclosing opportunities for future research. With that view, we selected several literature and investigated what attributes are incorporated in various systems to address wandering management. We draw an overview of the systems, focusing on technologies employed, underlying strategies or algorithms, scenarios or system goals and searched for overlapping or common grounds, along with challenges inferred from experimental results. We are going to list the technologies proposed or utilized in existing literature as well as real world devices.

The subsequent chapters elaborate on the above- mentioned points. While section II summarizes the methodologies of literature selection, Sections III and IV mentions the individuals and scenarios the systems are designed around. Sections V, VI, VII, and VIII consists of a survey on proposed or existing systems for wandering detection or management, built for indoor and outdoor scenarios, addressing various forms of wandering. Finally, we conclude in Section X, preceded by a discussion in Section IX.

\section{SeARCh Methodologies}

In this study, we aimed to gain insights on current methodologies of technological intervention and related challenges in the domain of wandering management, which will provide platform for future design opportunities. We selected 
twenty-three literature from scientific journal and conference publications. Additionally, we reviewed eight commercially available systems to explore technologies employed in real world scenarios. Initially, we used Google Scholar search to retrieve the pertinent literature. Subsequently we focused our search to specific journal and conference domains based on the preliminary search results and literature. In preliminary attempts of searching relevant literature, we employed 'dementia', 'Alzheimer's disease', 'wandering', 'wandering behavior', 'detection', 'classification', 'prediction', 'wandering patterns', 'algorithm', 'technology', 'design' and 'management' keywords in various combinations. We integrated, 'mild cognitive impairment', 'elderly', 'GPS', 'location', 'tracking', 'caregiver', 'assistive technology', and 'sensor' keywords in subsequent searches. We excluded literature concerning dementia diagnosis, solutions for dementia symptoms not related to wandering (i.e. memory improvement exercise), activity detection with no specific component for wandering detection, topics unrelated to technology in wandering management, and intervention methodologies.

\section{TARGET ACTORS}

Primary actors or users of the proposed systems are individuals suffering from various levels of dementia. Technological interventions may be selected based on patient's level of cognitive decline measured by medical scales (Global Deterioration Scale (GDS) or Reisberg Scale [7]). People with mild cognitive impairment, capable of independent living to some degree, may be equipped with system built to handle outdoor wandering. Patients with greater level of cognitive decline, confined to a secured indoor environment for their safety and well-being, are most likely to be assisted with systems built for indoor wandering management. Secondary actors or users of proposed systems are the caregivers (relatives or paid professional helpers) of dementia patients. In most systems, their role is to receive updates of patient status or notifications during critical situations. Some systems integrate emergency services (Law enforcement or medical services) as actors with approval from caregivers and enable their assistance to ensure patient safety.

\section{TARGET SCENARIOS}

Design of a system depends substantially on target scenario. This is evident in the variation of scenarios researchers selected, primarily to narrow down to one component or perspective of wandering and deal with the trade-off between simplicity and efficiency of a solution. There are two scenarios in broad spectrum regarding the location of wandering behavior: outdoor and indoor. When the patient is confined to a residence or care facility, it comes under the category of indoor wandering behavior. On the other hand, a patient traveling around much larger area (maybe around a city) falls under the radar of outdoor wandering behavior. Depending on whether the patient is inside or outside, the employed technologies may vary extensively. To identify and address wandering in the outdoor environment, several research studies have developed algorithms and GPS based solutions as non-pharmaceutical approaches and demonstrated promising results in terms of data output and human response [6] [8] [9] [10] [11] [12] [13]. Solutions proposed to tackle outdoor wandering considers travelling in larger areas by foot or by means of vehicular transportation (public and private). For example, Opportunity Knocks [14] (targeted for individuals with mild cognitive impairment) incorporates use-case for public transportation to improve independent life style. Reference [15] describes two scenarios - spatial disorientation (individual can not recognize surroundings and is unable to return to a known place) and goal-oriented disorientation (individual travels to an irrelevant place on purpose due to warped memory). Reference [8] considers speed of travel to differentiate between walking and riding motor vehicle. Wandering pattern detection in indoor ecology has been examined in studies both in technology domain [16] [17] and in medical field [18], [19], [20], [21], underscoring the significance of such an analysis. Indoor wandering monitoring systems deal with scenarios where patient attempts to leave residential or care facility unattended (elopement), moves around the facility or inside one room aimlessly following some patterns, leaves bed or room at usual sleeping hours or falls [22], [23].

\section{INDOOR WANDERING MANAGEMENT}

In this section, we discuss systems addressing wandering management in indoor environments, i.e. private residence or nursing homes. Tables I and II summarizes the described systems.

\section{A. Smart Home}

Doughty et al. [24] proposed placement of various sensors and actuators devices across the residence to manage wandering behavior. Wrist-worn devices are proposed to track sensor activation and to send alarms to actuators. For example, triggered by a door sensor, the wrist-worn device may send radio beacon to remote authorities indicating elopement. Software running on a local personal computer acts as the central management component. ID codes of devices are transmitted using FM radio pulse signal and source of transmission is located using high gain directional antennas.

\section{B. Smart Hospital}

A Smart Hospital system architecture is proposed by $\mathrm{Nu}-$ gent et al. [26]. In a hospital topology, active and passive RFID tags can be attached to objects and individuals, with RFID readers placed on doorways. Signals from local readers are aggregated by ward and floor level reader nodes and sent to a central IT server through a middleware (Application Level Event engine). To enhance the accuracy of the location data, the authors propose multi-modality, such as utilizing signals from mobile both Bluetooth and Wireless Local Area Network devices.

\section{Late Hour Wandering Detection}

Night time wandering, due to abnormal sleep pattern, could be hazardous for people with dementia. Supervision to such behavior requires modification to caregiver sleep routine, which consequently disrupts their daily life. With an aim to detect if patient is leaving bed, Masuda et al. [25] integrated a system composed of a mat-shaped step sensor which, if stepped on, gets activated, triggers an illumination system around the area, and sends out signal to a wandering alarm component. This 
TABLE I. A SUMMARY OF INDOOR WANDERING MANAGEMENT SYSTEMS PROPOSED TO AID DEMENTIA PATIENTS AND CAREGIVERS.

\begin{tabular}{|c|c|c|c|c|c|c|}
\hline & Year & Goal & Data & Sensors & Hardware & $\begin{array}{l}\text { Technology/ platforms/ } \\
\text { frameworks }\end{array}$ \\
\hline [24] & 1998 & $\begin{array}{l}\text { Activity and event detec- } \\
\text { tion (i.e mobility) }\end{array}$ & Multiple type & $\begin{array}{lr}\begin{array}{l}\text { Passive infrared } \\
\text { Inductive rensors, }\end{array} \quad \text { coupling } \\
\text { sensor, Identification } \\
\text { badge, Piezoelectric } \\
\text { sensor, Microphone, Mat- } \\
\text { shaped step sensors, Door } \\
\text { sensors, Thermostats } \\
\end{array}$ & $\begin{array}{ll}\text { Desktop computer, } & \text { Per- } \\
\text { sonal handy-phone } & \text { sys- } \\
\text { tem, Light source, Dis- } \\
\text { play device }\end{array}$ & - \\
\hline [25] & 2002 & $\begin{array}{l}\text { Detect night time wander- } \\
\text { ing }\end{array}$ & $\begin{array}{l}\text { Tag ID, } \\
\text { Reader ID }\end{array}$ & Step sensor & $\begin{array}{l}\text { Sensor signal receiver, } \\
\text { Lighting }\end{array}$ & $\begin{array}{l}\text { Personal handy-phone } \\
\text { system (PHS) }\end{array}$ \\
\hline [26] & 2003 & $\begin{array}{l}\text { Locate Wandering person } \\
\text { in a large facility }\end{array}$ & sensor activation signal & RFID & $\begin{array}{l}\text { RFID tag \& receiver, Mo- } \\
\text { bile device, Central server }\end{array}$ & $\begin{array}{l}\text { Application Level Event } \\
\text { (ALE) engine, Bluetooth, } \\
\text { WLAN, Bayesian Net- } \\
\text { work, Sequence Matching }\end{array}$ \\
\hline [23] & 2007 & $\begin{array}{l}\text { Detect night time wander- } \\
\text { ing }\end{array}$ & Sensor activation signal & $\begin{array}{lrr}\text { Bed occupancy } & \text { sensor, } \\
\text { Motion sensor, } & \text { Door } \\
\text { opening sensor } & \\
\end{array}$ & Wireless receiver & - \\
\hline [22] & 2011 & $\begin{array}{l}\text { Detect night time wander- } \\
\text { ing }\end{array}$ & - & $\begin{array}{l}\text { Ultra-wideband impulse- } \\
\text { radio(UWB-IR) }\end{array}$ & $\begin{array}{l}\text { UWB-IR generator, low- } \\
\text { noise amplifier (LNA), } \\
\text { digitizer, transmit/receive } \\
\text { antenna }\end{array}$ & - \\
\hline [27] & 2011 & Wandering prediction & $\begin{array}{l}\text { Location coordinates } \\
\text { Transponder } \\
\text { Date, Time }\end{array}$ & $\begin{array}{l}\text { Active Ultra Wideband } \\
\text { RFID }\end{array}$ & $\begin{array}{l}\text { RFID tag transponder and } \\
\text { sensor, Ethernet switch, } \\
\text { Network cable, Notebook } \\
\text { computer }\end{array}$ & Ubisense 2.0 software \\
\hline$[28]$ & 2015 & Wandering prediction & $\begin{array}{l}\text { Activity frequency and } \\
\text { time, Step count, Heart } \\
\text { rate, Location visit fre- } \\
\text { quency, Event frequency } \\
\text { and time }\end{array}$ & $\begin{array}{l}\text { Wrist worn activity sen- } \\
\text { sor (step counter), Heart } \\
\text { rate sensor, Switch sen- } \\
\text { sor, 3d-depth camera }\end{array}$ & Kinect & - \\
\hline
\end{tabular}

TABLE II. A SUMMARY OF EXPERIMENTS CONDUCTED TO EVALUATE INDOOR WANDERING MANAGEMENT SYSTEMS

\begin{tabular}{|c|c|c|c|c|c|}
\hline & Study type & Implementation & $\begin{array}{l}\text { Evaluation/ } \\
\text { Experiment }\end{array}$ & Experiment detail & Result \\
\hline [24] & No & No & No & - & - \\
\hline [25] & Clinical trial & Yes & Yes & System testing, 3 participants, 4 weeks & Detected wandering 30 times \\
\hline [26] & No & No & No & - & - \\
\hline [23] & Clinical trial & yes & yes & Control group experiment, 55 residence & Not reported \\
\hline [22] & User study & yes & yes & System testing, Detect scenarios & Detection rate $95 \%$ \\
\hline [27] & Clinical Trial & yes & yes & - & - \\
\hline [28] & No & No & No & - & - \\
\hline
\end{tabular}

component has a personal handy-phone system (PHS) terminal that sends warning notification and step sensor ID to caregiver PHS receiver. A solution proposed by Rowe et al. [23] is designed to alert caregivers only when patient leaves bed at night time, thus reducing the need for constant vigilance at late hours. The system consists of bed occupancy sensor, motion sensor, door opening sensor, wireless receiver and control panel running a software with specialized features. The bed occupancy sensor is an air bag connected to a transmitter, through an air pressure switch. When air pressure switch changes state (open when air pressure is low or close when pressure is high), the transmitter sends signal (off or on) to a remote receiver. Ota et al. [22] employ ultra-wideband impulse-radio (UWB-IR) to detect specific states of a patient, such as - static on bed, moving on bed, fall, wander inside room, get in or out of the room. UWB-IR is a non-obtrusive technology, capable of detecting nuances in movement from afar while preserving privacy and health. Distance of various objects from the sensors generates a range of received power delays. Moreover, movements (introducing new object on or near static objects) change the values of power delays, making them detectable.

\section{Prediction Frameworks}

Based on the wandering activities listed in Algase Wandering Scale (AWS) [29], a framework for predicting wandering behavior in indoor environment is proposed by Toutountzi et al. [28]. They propose to employ an assortment of sensors (Step counter, heart-rate sensor, door sensor, 3d-depth camera) to collect and compile data to detect some of the factors stated in AWS, such as increased aimless and repetitive walking or decreased sleep time. Active RFID (Radio Frequency Identification) generated data is used in a series of studies [20], [27], that aims to verify the role of tortuosity in movement data in predicting dementia. Reference [27] employs wrist-worn RFID transponders along with wall-mounted UWB (Ultrawide Band) sensors and a real-time location analysis software. To predict wandering in the Smart hospital environment, Nugent et al. [26] propose an event-based sequence matching prediction algorithm. The goal is to predict the next event in a sequence of events. Transitions from one event to another are extracted from previous data, with a score associated with each pair. For each new event in the sequence, score associated with the transition to the current event from the previous event is increased by a constant factor. Scores for all other transitions to the current event are decreased by a constant factor. When predicting an upcoming event, the highest scored transition from current event is selected.

\section{OUtDoor WANDERING MANAGEMENT}

In this section, we would discuss some systems where outdoor location data plays a central role in wandering man- 


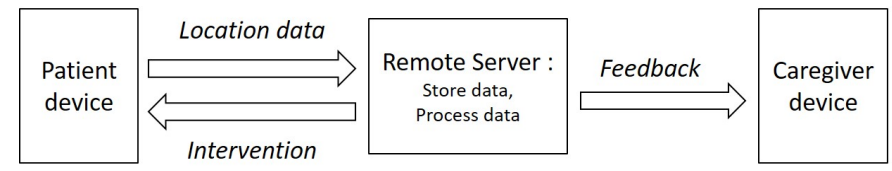

Fig. 1. General black-box model of outdoor wandering management systems. Overview of the proposed system architectures convey a general flow: acquire location data from patient, transmit location data to server, run calculations and transmit result to caregiver, and then transmit back intervention messages to patient. One or more components of this flow are present in the discussed systems. The differences lie in underlying technologies and frameworks, influenced by available devices at the time of the research.

agement. Tables III and IV summarizes the described systems. Vuong et al. [30] describe a general design in this regard. In majority of the systems, the central task is to detect the current location of the patient. Sensors embedded in a mobile device is carried by the patient. Location details of the carrier are sent to a remote server that runs wandering or anomaly detection applications and transmits intervention signals back to patient. Some systems include a caregiver device in ecology, to monitor patient status and receive notifications. Fig. 1 illustrates a general flow of the systems. Standard state-ofthe-art location detection, communication and network services are used for tracking and data transmissions. A non-exhaustive list of technologies includes Global Positioning System (GPS), Geographic information system (GIS), Global System for Mobile communication (GSM), Wi-Fi and Bluetooth. Collected tracking data may be stored in databases. How long the data should be stored, may depend on the goal. For example, if the data is collected to infer cognitive health by observing human behavior, then it need to be stored for a longer time, but if the goal is to predict or detect immediate wandering episodes or to estimate current location of the patient, the data can be discarded from memory after a shorter period.

\section{A. General Tracking Systems}

A GPS tracking system is proposed by Shimizu et al. in [31] built with a GPS receiver and a mobile phone carried by person with dementia and a remote personal computer. The GPS receiver retrieves location data (longitude and latitude coordinates) from GPS satellites and transmits it to the remote computer via the mobile phone over a mobile telephone network. The patient's location can be monitored by a caregiver through the computer. Calvo et al. developed a similar system [33] using an Android mobile device with 2G, 3G, Bluetooth and $\mathrm{Wi}-\mathrm{Fi}$ as data transmission mediums. To communicate patient's location to caregivers, they implemented a mobile social network engine LibreGeoSocial. Mulvenna et al. [43] developed a software COGKNOW to help dementia patients in independent living. A tablet computer works as a hub for sensors placed at doors and furniture at home, while outside, a mobile phone collects Geo-location data using GPS. A server is connected to the system that stores data accessible by caregivers through a web interface.

\section{B. Destination Oriented Travel}

Opportunity Knocks, a solution proposed by Patterson et al. [14], was built to aid in destination-oriented travel where person with dementia needs to travel around a city area using public transport. While traveling using public transportation, the patient carries a sensor beacon and a mobile phone. Sensor beacon collects GPS data and transmits to a mobile phone by Bluetooth. Based on current location, the application running on the mobile device, shows images of potential destinations (selected from frequently visited places by the patient). The mobile phone, working as a network access point, sends data to a remote server using GPRS network. Based on location (safe or unsafe route), the server sends back intervention data (i.e. bus route to destination) to the patient phone, which produces audio-visual assistance and alerts. When user selects a destination, bus routes are suggested, and instructions regarding the next course of actions are conveyed (i.e. bus stop to board or get off). As user progresses along a route, user locations are processed to determine if he is on the correct path. If user diverge from suggested route, warning prompt is produced, and instructions are updated to bring back user to correct route. The system also has provisions to differentiate between incorrect travel route and purposeful new route.

IRoute system [37] deploys Belief-Desire-Intention (BDI) architecture, to predict travel in one or more potential travel routes, depending on one or more destinations. Previous travel information is leveraged to predict routes to a goal destination. The system tracks person with dementia in real-time and updates predictions according to location changes. Deviation from predicted route is considered anomalous behavior. As an intervention technique, correct route is provided to coerce person with dementia to follow correct path. Failure to comply with the guidance triggers system to notify caregivers. Running on a GPS equipped mobile phone, the BDI agent is responsible for route prediction using user input (list of travel locations and activities, frequency of occurrences, start times and destination locations) and routes stored from previous travels (a set of time stamps and GPS location points to a destination location).

\section{Safe-Zone or Geo-fence Centered Systems}

Some systems employ algorithms devised around safe-zone paradigm where person with dementia is secured if his or her location is inside a predefined virtual geographical fence (geofence) or zone. In this section, we discuss systems that utilizes this idea. Various ways are described for defining safe zones. A circular safe zone could be defined with a small radius, initially encompassing only patient's residence and adjustable when needed [8]. The center could be determined by where patient's tracking device is charged for a extended period of time. The geo-fence could be centered at home with a radius extending to the farthest frequented location selected upon interview with person with dementia and caregiver [38]. Author in [41] defines Home-zone and Secure-zone, where patient lives in Home-zone (a point element on map) and Secure-zone is polygon shaped area surrounding Home-zone. Multiple zones could also be defined to indicate safety status of person with dementia. A set of discrete locations (home, close-to-home, far-from-home) could be derived from GPS location data. The zone borders can be drawn manually on a map application, or learned using heuristic, statistical clustering or Bayesian method [15]. In [40], a set of points are selected as secured places (i.e. home, relative's house), called Hot-Spots. Separate circles are defined, centered at each Hot-Spot, to mark zones. The zones are defined in a similar way as [39] - familiar area, caution area and completely unfamiliar area. A series of safe 
TABLE III. A SUMMARY OF OUTDOOR WANDERING MANAGEMENT SYSTEMS PROPOSED TO AID DEMENTIA PATIENTS AND CAREGIVERS



zones are identified automatically in [42], by mining travel data and detecting most frequented places. Gaussian Distribution is utilized to normalize numerous location points for defining a precise safe zone. Lost-zones [42] can be formulated based on locations from where user takes longer time to come back home. Zone thresholds are gradually learned from accumulated travel data over time. Centered at the same point, [39] drew two circles on a map to define zones. The area inside the smaller circle is the safe-zone; the area between the larger and the smaller circle is considered a warning zone; area outside of the larger circle is considered unsafe.

iWander, an mobile application developed by Sposaro et al. [8], leverages technology and services embedded in a mobile phone device, based on the reasoning that a person carries a mobile phone outdoors at all times. The application runs in the background and tracks location and weather data. Divergence from patients' regular routes or travel outside of a defined safe zone activates the application, notifying the caregiver and providing correct route direction to patient. It also considers the speed with which the patient is traveling, to determine automobile travel using Haversine formula [44]. Furthermore, a wandering detection algorithm is formulated that applies Bayesian Networks model. It calculates the conditional probability of occurrence of wandering, given the age of patient, dementia stage of patient, time of day, time outside the safe zone and weather condition. Support vector machine followed by a nonlinear regression is used to classify regular and abnormal behavior.

The system designed by Wan et al. [34] and [35] consists of specialized service oriented interconnected software, running 
(IJACSA) International Journal of Advanced Computer Science and Applications,

TABLE IV. A SUMMARY OF EXPERIMENTS CONDUCTED TO EVALUATE OUTDOOR WANDERING MANAGEMENT SYSTEMS

\begin{tabular}{|c|c|c|c|c|c|c|}
\hline & Algorithm goal & $\begin{array}{l}\text { Intervention/ } \\
\text { Notifica- } \\
\text { tion }\end{array}$ & Prototype & Study type & Experiment detail & Result \\
\hline$[31]$ & Location detection & None & Yes & User study & $\begin{array}{l}\text { Feasibility study of GPS de- } \\
\text { vice }\end{array}$ & $\begin{array}{l}\text { GPS device is feasible if data } \\
\text { is collected sporadically }\end{array}$ \\
\hline$[32]$ & Tracking & Notification & Yes & User study (1 participant) & $\begin{array}{l}\text { Tracking capacity and rescue } \\
\text { time measurement }\end{array}$ & $\begin{array}{l}60 \text { meter detection radius, } \\
\text { mean rescue time } 13.1 \text { min- } \\
\text { utes }\end{array}$ \\
\hline$[14]$ & $\begin{array}{l}\text { Movement behavior learning, } \\
\text { Anomaly detection, Assistive }\end{array}$ & Intervention & Yes & User study (1 participant) & System test with scenarios & Successful \\
\hline$[33]$ & Tracking & Both & No & - & - & - \\
\hline [8] & $\begin{array}{l}\text { Movement behavior learning, } \\
\text { Anomaly detection }\end{array}$ & Both & Yes & Proposed clinical trial & - & - \\
\hline $\begin{array}{l}{[34],} \\
{[35]}\end{array}$ & Tracking & Notification & Yes & Clinical trial (52 participants) & $\begin{array}{l}\text { User satisfaction question- } \\
\text { naire, usability test }\end{array}$ & $\begin{array}{l}92 \% \text { task completion, } 75 \% \\
\text { positive usability }\end{array}$ \\
\hline$[36]$ & Location detection & Notification & Yes & User study ( 9 participants) & System test & $\begin{array}{l}\text { Minimum } 15 \text { second sound } \\
\text { data for } 100 \% \text { accuracy }\end{array}$ \\
\hline$[37]$ & $\begin{array}{l}\text { Movement route prediction, } \\
\text { Anomaly detection, Assistive }\end{array}$ & Both & Yes & $\begin{array}{l}\text { Clinical trial (one participant } \\
\text { with } \mathrm{MCI} \text { ) }\end{array}$ & $\begin{array}{l}\text { Tested single and multiple } \\
\text { destination prediction scenar- } \\
\text { ios }\end{array}$ & $\begin{array}{l}\text { System works for one exam- } \\
\text { ple }\end{array}$ \\
\hline$[15]$ & $\begin{array}{l}\text { Movement behavior learning, } \\
\text { Anomaly detection, Assistive }\end{array}$ & Both & Yes & - & - & - \\
\hline$[38]$ & $\begin{array}{l}\text { Online location tracking, } \\
\text { Zone status detection }\end{array}$ & Both & No & - & - & - \\
\hline [39] & Tracking, Fall detection & Both & No & - & - & - \\
\hline [40] & Tracking, Anomaly detection & Notification & Yes & - & $\begin{array}{l}\text { Measured performance accu- } \\
\text { racy of eleven features }\end{array}$ & $\begin{array}{l}\text { Accuracy, false positive and } \\
\text { false negative evaluated. }\end{array}$ \\
\hline$[41]$ & $\begin{array}{l}\text { Movement behavior learning, } \\
\text { Anomaly detection }\end{array}$ & Notification & Yes & Clinical trial & $\begin{array}{l}\text { Usability test, Interview, } \\
\text { Questionnaire }\end{array}$ & $85 \%$ positive usability \\
\hline$[42]$ & Movement behavior learning & Both & No & - & - & - \\
\hline
\end{tabular}

on a patients' device, a caregiver device, and a data server. The data server is equipped with authorized web service, to securely track patient on a map, access patient history data, create safe zones and perform related tasks, register patients and caregivers, and update profile information. Alerts are sent to caregiver in case patient is outside of the safe zone.

LaCasa [15] employs Partially Observable Markov Decision Process (POMDP), to learn known locations of person with dementia, and using that knowledge, detects anomalous travel behavior and provide assistance when needed. A safezone is created where the patient resides more than 21.5 hrs./day or there is Wi-Fi connectivity at a known network. A list of known locations is stored in a server. The patient mobile device runs an application, InCense, built for behavioral data collection. When InCense fails to detect a safe-zone WiFi (or cellular) access point, it is triggered to collect location data and initiate intervention methods such as displaying stored images of known locations, playing audio prompt, or sending a reminder SMS.

A solution is propo sed by Ogawa et al. [32], that utilizes Personal Handy-phone System (PHS) transmitter/receiver network to create a safe-zone. The caregiver is notified via a mobile device if patient is outside the threshold of the safe zone (not within 100 meters of home). Matsuoka et al. [36] uses a similar communication and sensor/receiver deployment mechanism but with a different patient device. Wandering outside a predefined safe zone triggers the patient device to record environmental sound and to send to a server computer along with location data, which the server transmits to caregiver device.

Yuce et al. in [38] propose a social network of caregivers (CaregiverNet), to search for a wandering patient. A wristwatch tracker is worn by the patient that collects location data and sends it to a remote server periodically. Caregivers should also carry a smart phone that has a communication management application running on it. An auto intervention mechanism (Call-based Supervision) is employed where a Novoice communication GSM call is placed through tracker, to inquire patient status. Patient can ensure a safe status by placing a similar call through the tracker. Failure to get the safety response call from patient triggers the system to notify all registered caregivers, asking confirmation if patient is reachable. Negative response indicates patient is wandering. Wandering state triggers system to increase frequency of location update from patient tracker, to retrieve all phone numbers of registered caregivers and to send emergency message with patient location. Then it periodically sends the current location of patient to the caregivers, who agrees to volunteer in the search, and eventually stops when a 'FOUND' signal (indicting person with dementia is safe) is received from any caregiver.

Photo or images could be utilized as a data type in locating a potentially wandering patient (Ko et al. [39]). A camera equipped smart phone, attached to the body of person with dementia, takes environmental images periodically and sends to a remote cloud server, along with GPS location and time stamp data. Frequency of collecting images can be customized to save battery power. Even if GPS signal is lost in an indoor environment, the system continues to transmit images of the environment. Stepping outside a safe-zone triggers the system to play a prerecorded intervention audio message to patient, suggesting him/her to return to a known place. Caregivers are also notified via phone call and lack of response from caregivers triggers the system to notify emergency medical services.

IP Multimedia Subsystem (IMS) architecture is utilized to model a system suitable for wandering management by Moreno et al. [40]. They implement IMS Presence Service where 'Presentities' are patients, whose status are make known by the PUBLISH and NOTIFY methods to caregivers ('Watchers' of Presence Service), who are registered by SUBSCRIBE 
method. The 'Presence' entities of the model (GPS location, safe-zone information, speed of travel and timestamps) are sent from a mobile phone to a Presence server.

Another system that employs safe-zones is developed by Batista et al. [41]. An application runs on a GPS equipped smart phone, that collects location data and sends three consecutive locations as XML message format to a remote server at definite time intervals. To save battery life, data transmission frequency can be customized according to day, night and emergency period. Moreover, data is only collected if some movement is detected by smart phone accelerometer. A website enables caregivers to create and access patient profile, set parameters and monitor alarms generated by anomalous behaviors such as venturing outside safe-zone, odd-time movement, no movement or high-speed movement, and system failures.

With an aim to increase independence and privacy of person with dementia and reduce their dependence on caregiver, $\mathrm{Ng}$ et al. [42] designed a portable GPS device. To keep the user-interface simple, only two buttons are visible on the devices LCD display. The 'Home button' invokes a visual compass aiding the patient to find his own way home. In case the patient needs assistance, patients' current location is sent to caregiver pressing the 'Alert button'. The authors also formulated an algorithm where Safe-zone and lost-zone mechanisms are employed to assist in detecting wandering behavior. For 30 days, the system collects users "GPS footprint". In the learning phase, the algorithm starts to refine its parameters and to make decisions. A 20 days data window is used to update the parameters further. Distances from home and displacement times are statistically learned as parameters. Exceeding these threshold values would be considered as abnormal elopement behavior. In case of abnormal behavior, the system would generate audio and visual intervention for the user. MartinoSaltzman movement patterns [21] and framework developed by [16] are utilized to identify aimless walking.

\section{ALGORITHMS}

Several studies focus on formulating detailed algorithms to detect wandering. In this section, we explore three algorithms for detecting spatial disorientation and five algorithms to distinguish the four geographical patterns proposed by MartinoSaltzman et al. [21] to detect wandering behavior. Table V summarizes the described algorithms.

\section{A. Anomaly Detection with Zone Boxes}

Chang et al. [13] formulates an algorithm to detect anomalous behavior from travel paths or trajectories. Travel trajectory is represented by a series of rectangular boxes, each constituting a sub-trajectory (Fig. 2). To establish a trajectory that depicts normal behavior, a history of trajectories is utilized to find overlapping areas. A weight is assigned to each area or region, depending on the number of overlapped boxes, thus creating a weighted trajectory (Fig. 3). This weighted trajectory and probability are used to evaluate new trajectories to decide if they represent anomalous or wandering behavior.

\section{B. Anomaly Detection with Next Location Prediction}

Vuong et al. [30] proposes an extension (Adaptive Confidence Estimation) to 'next location prediction' algorithm [47],

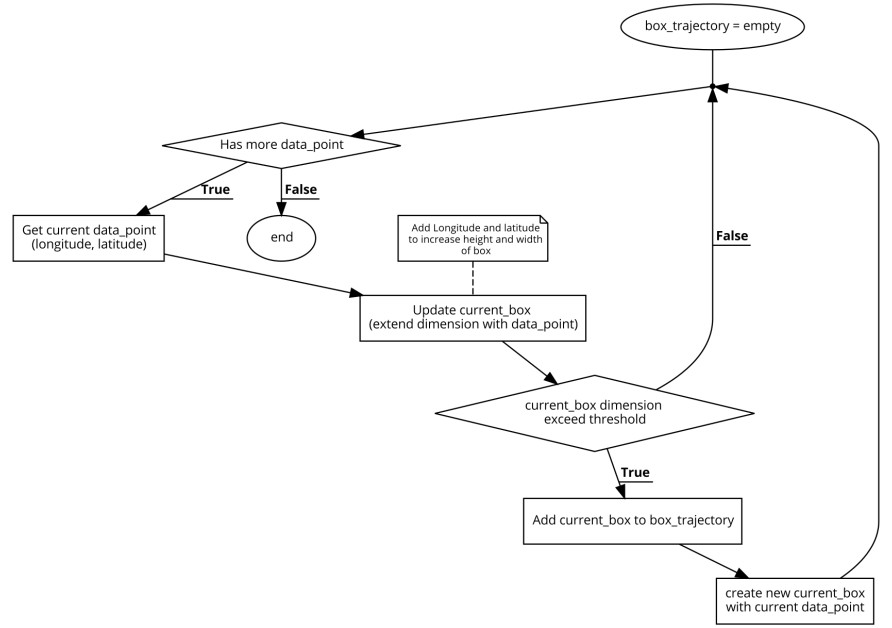

Fig. 2. Chang et al. [13] formulates an algorithm to detect anomalous behavior from travel paths or trajectories which are represented using boxes. For a subtrajectory, top and bottom sides of a box are maximum and minimum latitudes, left and right sides are maximum and minimum longitudes. To create and update a box, dimension thresholds are set, and incoming location coordinates are used to update the length of the sides of the box. If any one of the sides exceeds respective threshold, that box is added to trajectory. A new box is created for upcoming location points and the procedure is repeated.

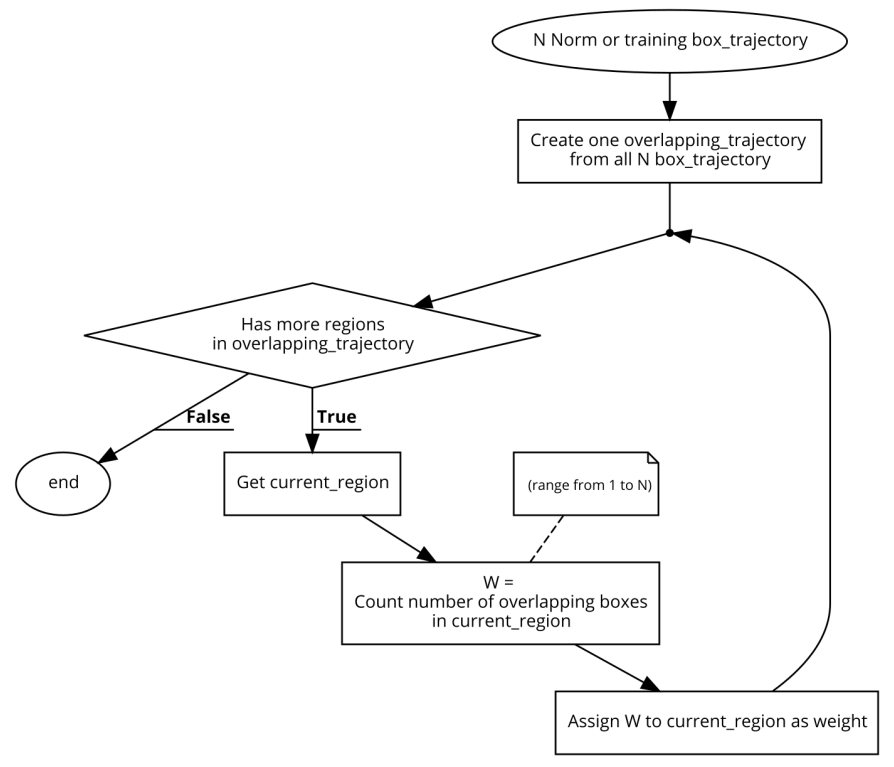

Fig. 3. Create Weighted Trajectory (Chang et al. [13]) using box representation to subsequently detect anomalous behavior from travel paths or trajectories

to utilize it in wandering management scenarios. The goal is to predict anomalous behavior as soon as possible. In [47], given a series of locations, the next location or state is predicted with a confidence value. A 'confidence counter' associated with that series of locations, keeps count of the correct predictions or prediction confidence. The counter value is increased by 1 if predicted result is correct and decreased by 1 otherwise. If the value exceeds a threshold, the prediction is 'deliverable' or reliable. Authors from [30] updates the 'confidence counter' based on how frequently a location is visited or an event occurs. They propose to reduce confidence levels in a weighted manner rather than at a flat rate; incorrect prediction associated 
TABLE V. ALGORITHMS DEVELOPED FOR WANDERING BEHAVIOR DETECTION IN TRAVEL DATA OF DEMENTIA PATIENTS

\begin{tabular}{|c|c|c|c|c|c|c|c|c|}
\hline & Year & $\begin{array}{l}\text { Indoor/ } \\
\text { outdoor }\end{array}$ & Sensors & Data & Algorithm & Study type & Evaluation & Result \\
\hline [13] & 2010 & Outdoor & GPS & $\begin{array}{l}\text { Sequence of loca- } \\
\text { tions (latitude, lon- } \\
\text { gitude), Time }\end{array}$ & $\begin{array}{l}\text { Real time deviation or } \\
\text { anomaly detection with } \\
\text { Box trajectory, Move- } \\
\text { ment behavior learning }\end{array}$ & $\begin{array}{l}\text { Clinical trial (8 par- } \\
\text { ticipants) }\end{array}$ & Precision, recall & $\begin{array}{l}\text { Precision } .90, \text { re- } \\
\text { call } .95 \text {, Computa- } \\
\text { tion time } 15.1 \mathrm{~s} \text { to } \\
22.7 \mathrm{~s}\end{array}$ \\
\hline$[30]$ & 2011 & Indoor & - & $\begin{array}{l}\text { Sequence of loca- } \\
\text { tions (latitude, lon- } \\
\text { gitude) }\end{array}$ & $\begin{array}{l}\text { State predictors with } \\
\text { confidence counter } \\
\text { (CC), Adaptive } \\
\text { Confidence Estimation, } \\
\text { movement behavior } \\
\text { learning, next location } \\
\text { prediction, anomaly } \\
\text { detection }\end{array}$ & $\begin{array}{lr}\text { Augsburg } & \text { Indoor } \\
\text { Location } & \text { Tracking } \\
\text { Benchmarks }\end{array}$ & Accuracy & Accuracy .88 \\
\hline [16] & 2011 & Indoor & RFID & $\begin{array}{l}\text { Discrete locations, } \\
\text { Time }\end{array}$ & $\begin{array}{l}\text { Movement pattern de- } \\
\text { tection (Repeated loca- } \\
\text { tion count) }\end{array}$ & $\begin{array}{l}\text { Clinical trial data } \\
\text { set from [19] (1 par- } \\
\text { ticipant) }\end{array}$ & $\begin{array}{lr}\text { Classification } & \text { and } \\
\text { comparison } & \text { with } \\
\text { empirical data } & \\
\end{array}$ & Consistent results \\
\hline [6] & 2012 & Outdoor & GPS & $\begin{array}{l}\text { Sequence of loca- } \\
\text { tions (latitude, lon- } \\
\text { gitude), Time }\end{array}$ & $\begin{array}{l}\text { Movement pattern de- } \\
\text { tection (Episode Seg- } \\
\text { mentation with Vector } \\
\text { Angles) }\end{array}$ & $\begin{array}{lll}\text { User } & \text { study } & (100 \\
\text { traces }) & & \end{array}$ & $\begin{array}{l}\text { Area Under ROC } \\
\text { Curve }\end{array}$ & $\begin{array}{l}\text { Detection rate } 90 \% \text {, } \\
\text { False alarm rate } 5 \%\end{array}$ \\
\hline [17] & 2014 & Indoor & RFID & $\begin{array}{l}\text { Discrete locations, } \\
\text { Time }\end{array}$ & $\begin{array}{l}\text { Movement pattern de- } \\
\text { tection, Machine learn- } \\
\text { ing and Ad hoc ap- } \\
\text { proaches }\end{array}$ & $\begin{array}{l}\text { Clinical trial data } \\
\text { set from [19] (5 par- } \\
\text { ticipants) }\end{array}$ & $\begin{array}{l}\text { Precision, recall, la- } \\
\text { tency, specificity, F1 } \\
\text { measure }\end{array}$ & $\begin{array}{l}\text { Ad hoc algorithm } \\
\text { performed better } \\
\text { than ML, Random } \\
\text { Forest best in ML }\end{array}$ \\
\hline [12] & 2015 & Both & GPS & $\begin{array}{l}\text { Sequence of loca- } \\
\text { tions (latitude, lon- } \\
\text { gitude) }\end{array}$ & $\begin{array}{l}\text { Cycle and direction } \\
\text { analysis in travel } \\
\text { trajectory, Wandering } \\
\text { detection }\end{array}$ & $\begin{array}{lr}\text { User } & \text { Study } \\
\text { (wandering } & \text { data } \\
\text { set), SIMPATIC } \\
\text { clinical trial data set }\end{array}$ & $\begin{array}{l}\text { Detect cycles, direc- } \\
\text { tion change in two } \\
\text { data sets }\end{array}$ & $\begin{array}{l}\text { Wandering data set } \\
\text { has more cycles \& } \\
\text { direction change }\end{array}$ \\
\hline [45] & 2015 & Indoor & $\begin{array}{l}\text { Magneto } \\
\text { meter }\end{array}$ & $\begin{array}{l}\text { Acceleration, Orien- } \\
\text { tation }\end{array}$ & $\begin{array}{lr}\begin{array}{l}\text { Movement } \\
\text { detection } \\
\text { speed and attern } \\
\text { (movement }\end{array} \\
\text { pattern) } & \end{array}$ & $\begin{array}{l}\text { User Study (5 par- } \\
\text { ticipants) and clini- } \\
\text { cal trial ( } 2 \text { partici- } \\
\text { pants) }\end{array}$ & $\begin{array}{l}\text { Recall, latency com- } \\
\text { parison with two al- } \\
\text { gorithms }\end{array}$ & $\begin{array}{lr}\text { Average } & \text { recall } \\
83.44 \%, & \text { Latency } \\
12.8 \mathrm{sec} & \end{array}$ \\
\hline [46] & 2016 & Both & RFID & $\begin{array}{l}\text { Continuous coordi- } \\
\text { nates, Time }\end{array}$ & $\begin{array}{l}\text { Movement pattern de- } \\
\text { tection (Grid, episode } \\
\text { segmentation, travel ef- } \\
\text { ficiency, loop detection) }\end{array}$ & $\begin{array}{l}\text { Clinical trial } \\
\text { participants) }\end{array}$ & Recall, precision & Accuracy $90 \%$ \\
\hline
\end{tabular}

with more frequent locations are penalized more compared to less frequented locations.

\section{Wandering Detection with Cycles and Angles in Trajectory}

Batista et al. utilized location, temporal and acceleration data to formulate wandering detection algorithms. In [10], they hypothesized that randomness is an integral part of wandering behavior and concluded that short-length cycles in a travel trajectory infers to wandering. Building on their theory and centrality measure [48], they proposed a graph representation of trajectory paths and deduced that frequency of nodes in subgraphs can be used to detect wandering [10]. In a subsequent approach [12], the authors formulated two different algorithms to identify wandering segments in trajectory data. First, they selected a rectangular territory from the previously collected GPS data and divided it into nodes. In the first algorithm, they used the Schwarcfiter and Lauer's algorithm [49] to produce a set of cycles from a graph (JGraph from Java library). The second algorithm utilizes the JAMA (Java Matrix Package) to implement adjacency matrix, that stores the graph information and computes small-length cycles. To detect the direction and orientation of the individual, the method proposed by [6] was used.

\section{Temporal Episodic Approach}

An ad-hoc algorithm (Fig. 4) was devised by Vuong et al. [16], to detect and automatically classify Martino-Saltzman [21] patterns in movement trajectories. Time and location data utilized in the experiments are collected using RFID activity monitoring system in a different study conducted in
[19], [20]. The input to the algorithm is spatiotemporal data, where locations are specific spots at an indoor environment and temporal data are time instance or time spent at each location. The authors assumed that time spent to move directly between pairs of consecutive locations are constant and is set as hyper parameters of the algorithm.

\section{E. Utilization of Vector Angles}

Algorithm proposed by Lin et al. [6] (Fig. 5) uses the angle between two travel trajectories to detect wandering. The algorithm aims to detect wandering patterns based on sharp direction changes and segments. The authors considered only lapping and pacing patterns [21] as indicators of wandering.

\section{F. Deterministic Algorithm}

Vuong et al. [17] formulated a deterministic algorithm (Fig. 6) that classifies snippets in travel episodes as direct, lapping, pacing or random [21]. Travel episodes, composed of a sequence of locations and defined by start and stop locations, are segmented by a module that considers stopping threshold, maximum direct travel time, and wandering offset time. Locations in a trajectory represents discrete locations in a living facility as opposed to continuous location data. Extracted episodes are inputs to a deterministic tree-based algorithm, that detects the travel patterns [21] contained in an episode.

\section{G. Machine Learning Approaches}

In [17] the authors employed eight Machine Learning algorithms - Naive Bayes, Multilayer Perceptron, Random 


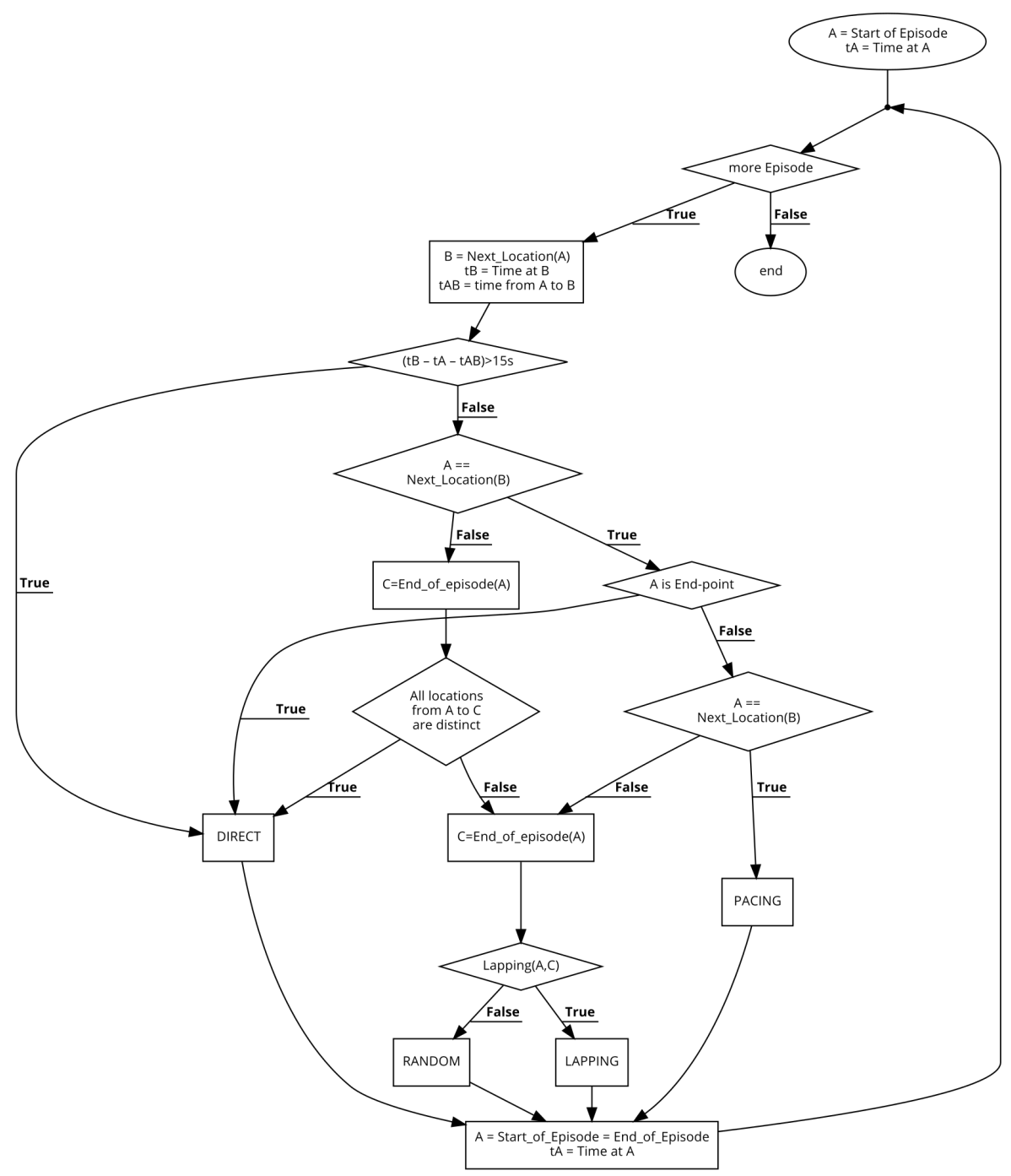

Fig. 4. Wandering pattern identification algorithm proposed by Vuong et al. [16] that recognizes the Martino-Saltzman patterns (Direct, Random, Pacing, and Lapping) [21] in the movement trajectory of a dementia patient. The complete spatial movement trajectory can be divided into 'episodes' by time. Each episode consists of locomotion (time spent to move from a source location to a destination location) and non-locomotion (time spent at the destination location) phases. An episode is formed with one or more consecutive movements (moving from one location to an immediate next location). The end location of one episode is the start location of the next episode. A location is the end-point of an episode, if the duration of the episode exceeds 5.41 minutes at that location or time spent at a location is more than 15 seconds. A pacing episode should include at least three movements between same two locations, whereas a lapping episode should include at least two circular movements among at least three locations.

Forest, Bagging, Support Vector Machine, K Nearest Neighbor, Logistic Regression, and Pruned Decision Tree (C4.5), on data from [19] [20], in Weka environment, to classify travel episodes as direct, random, lapping or pacing. Entropy, number of repeated locations, number of repeated travel directions, and number of opposite travel direction pairs are used as attributes.

\section{H. Patterns from Inertial Sensor Output}

Vuong et al. [45] use inertial sensor signals to distinguish the four wandering patterns [21] in movement. An accelerometer is utilized to calculate the acceleration of the patient to detect locomotion. A magnetometer component is used to measure the orientation data of the patient. In ideal cases, the system should output different types of orientation signals for the four patterns To remove noise created by fluctuations, they use scalar quantization to clamp a range of angular values to discrete values.

\section{Grid World Approach}

Kumar et al. [46] redefined the four Martino-Saltzman movement patterns (direct, lapping, pacing, random) [21] to make them fit into a square grid representation of the environment. They distinguish among the patterns using grid and subpath interaction style, path efficiency, number of loops in the path, and area within a loop. A movement trajectory is divided into non-locomotion (no motion for more than sixty seconds) and locomotion segments. Each locomotion segment is an 'episode' which is divided into 'looping' (longest continuous segment which intersect with itself) and 'non-looping' (longest continuous segment which does not intersect with itself) segments. Non-looping segments are labeled 'direct' or 'random' based on travel efficiency. The looping segments are labelled 


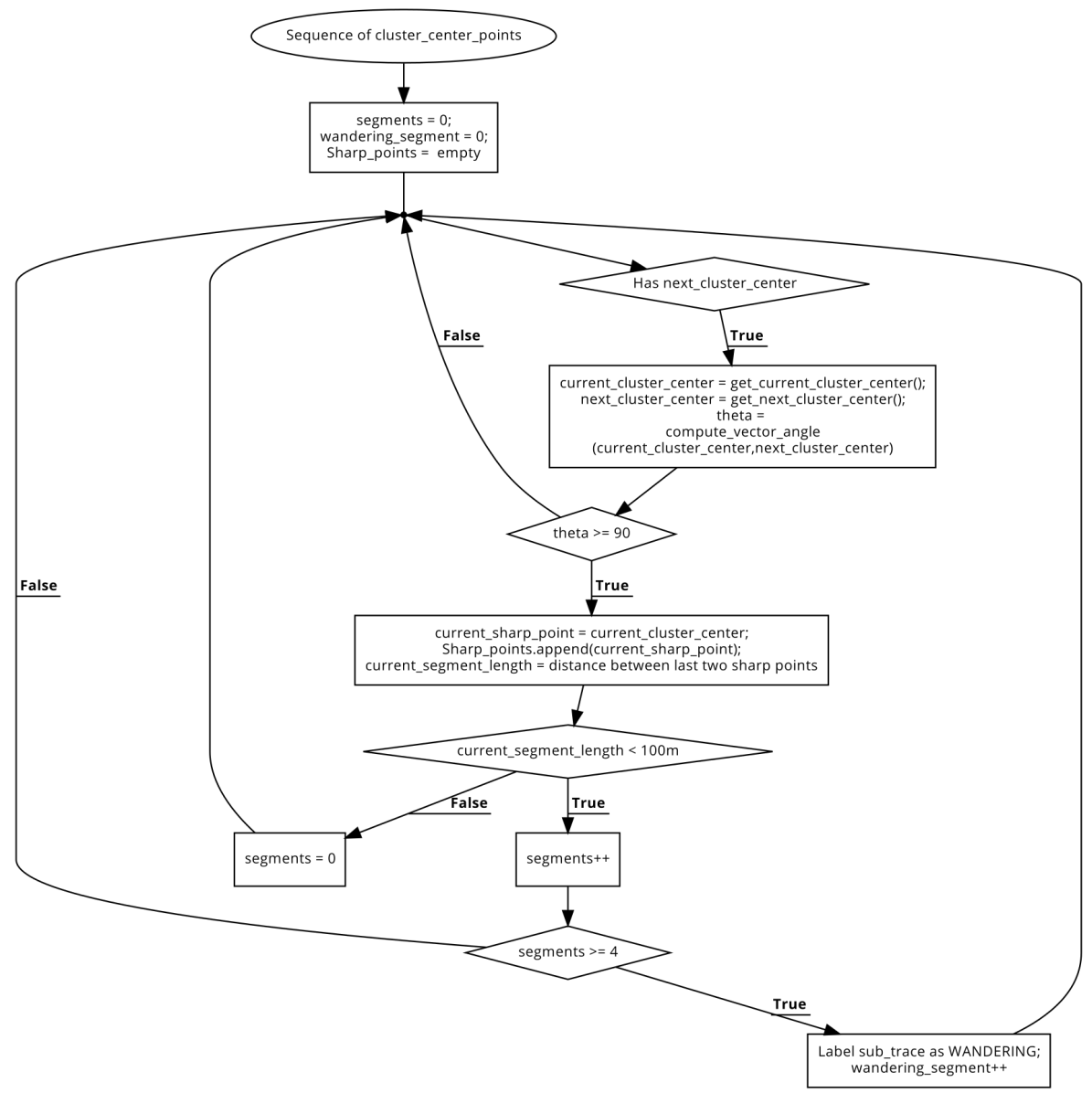

Fig. 5. $\theta W \_D$ wandering detection algorithm proposed by Lin et al. [6] that indicates if a movement trajectory of a dementia patient contains wandering episodes. 'Sharp points' are positions in the travel trajectory where the angle is at least 90 degrees. The authors defined wandering as - "a loop-like travel, with each loop that consists of a series of trace segments, clamped by two adjacent sharp points within a given distance range".

'lapping' or 'pacing' if there are two or more consecutive, slightly overlapping loops. Enclosed area that exceeds the minimum area possible with a given segment length indicates a lapping pattern.

\section{Commercial Devices}

Wandering behavior, requiring an extensive amount of supervision, inspires an assortment of assistive commercial products [50] that leverages current technologies. Trax [51] is a real time GPS tracker that transmits patient's location, speed and orientation data through cellular network to a smart phone application. When patient steps out of predetermined safe zones or Geofences at specific times, a warning notification in issued to caregivers. Safe Link [52] is another real time GPS tracker that periodically sends location data via internet to a cloud based remote server which is accessible to caregivers via internet through a website. PocketFinder [53] leverages multiple locator technology, transmitting location information every 60 seconds to a mobile application. Trajectory data is stored for 60 days. Equipped with a GPS transmitting data every 4 minutes, Mindme locator device [54] is used to track its carrier on-line using a website. It is equipped with multi-network SIM card, widening its connectivity range with multiple cell networks. In GPS Smart Sole [55], GPS technology is embedded in a shoe sole to be put inside a shoe.
GPS data is transmitted real time to a remote server via cellular network and compiled into a trajectory history report, to be accessed by caregivers via smart phone application or desktop computer browser. Connected to a smart phone application, iTraq [56] utilizes multiple technology to collect nuanced location data. Data can be accessed via internet through smart phone application. AngelSense [57] device keeps track of the patient in both indoor and outdoor environments, learns travel patterns, and alerts the caregiver in case of increased speed, delays or unfamiliar location. Project Lifesaver [58] is a radio frequency enabled tracking system that allows remote tracking of the patient, consisting of a transmitter worn by the patient and a receiver device for the caregiver. We summarize features of the mentioned devices in Table VI.

\section{DISCUSSION}

Technology could play a beneficial role in aiding individuals with dementia if employed in a pragmatic way. For example, if the device design or usage mechanism is too complicated for the patients, it might cause hindrance in their daily lives. In a participatory design study conducted by Robinson et al. [59], several factors have been pointed out by users (dementia patients and their caregivers) regarding the purpose of technology in wandering management. Technology should help prevent patients from getting lost, reduce caregiver 


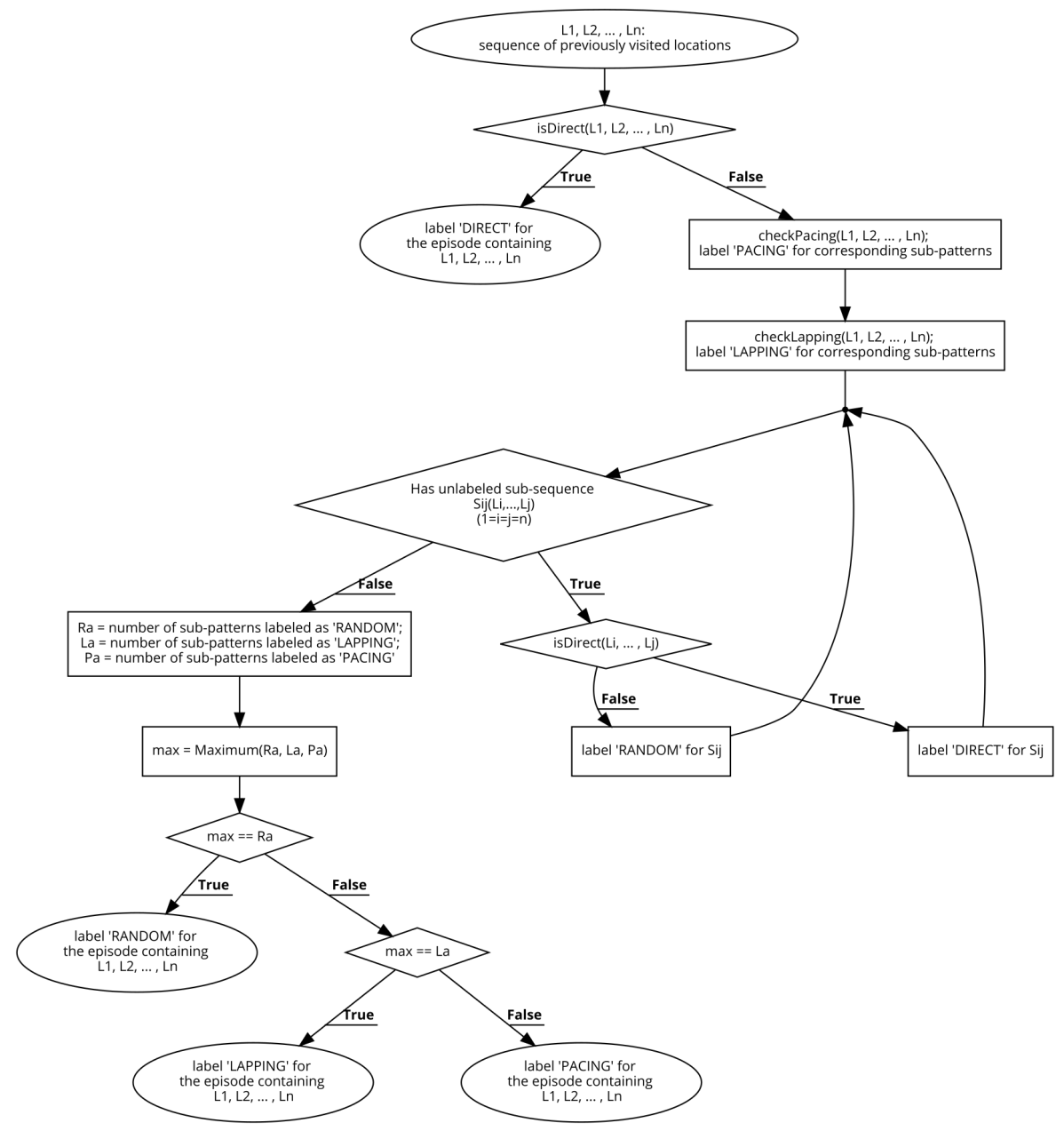

Fig. 6. Deterministic algorithm proposed by Vuong et al. [17] to detect and label the four wandering patterns (Direct, Random, Pacing, and Lapping) [21] in movement trajectory data of patients with dementia. Three sub-modules are employed to detect and mark direct, lapping, and pacing sub-sequences within an episode; any other sub-sequence is marked as random.

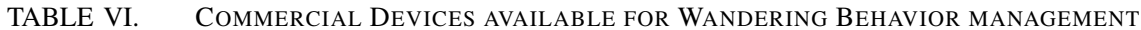

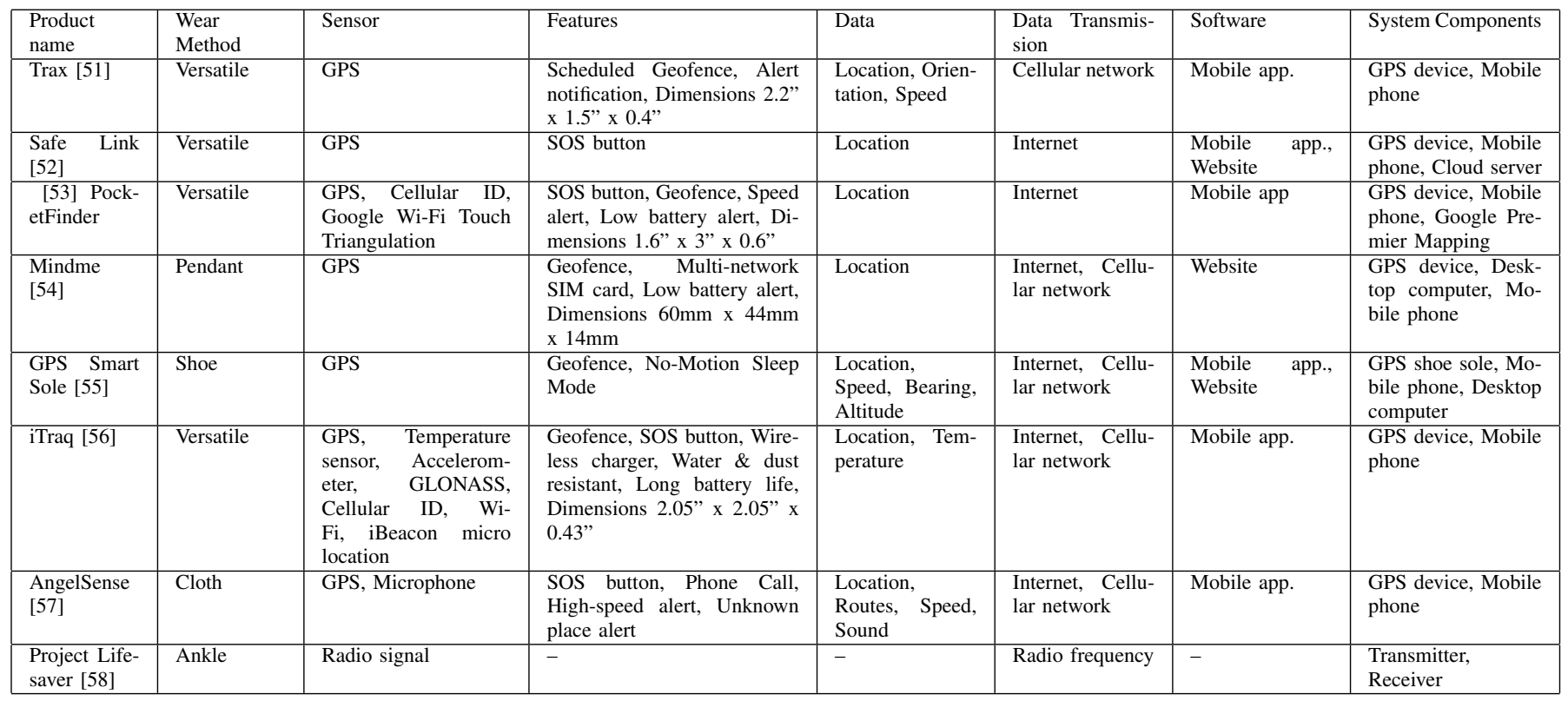


anxiety and abrupt disruption in caregiver routine, aid in usual activities, as well as promote independence and confidence. Technology could aid patients to help themselves, without seeking help from others, as well as have the provisions to start communication in emergency situations and initiate a rescue. In another study, McCabe et al. [60] focused on how a GPS embedded device could assist dementia patients in coordination of travel or movements and prevent wandering related hazards and they received similar feedback from users. The objectives are to increase quality of life by promoting independence, to reduce risks, stress and burden of vigilance, to increase safety and security, and to provide assurance of being found if lost, thus giving more freedom to travel to unfamiliar places. The challenge is to incorporating technology in everyday life so that they do not disrupt regular activities along with freedom and privacy [59]. To always remember and maintain to carry device is another challenge to be observed while designing a new device [59].

\section{A. Design Suggestions from User Studies}

We want to analyze if the systems described above comply with the facts revealed in user centric design studies by Holbo et al. [61], Robinson et al. [59], McCabe et al. [60], and Wan et al. [62].

1) Mobile Device: We notice, all solutions we discussed so far, requires a person with dementia to carry a mobile device, may it be a mobile smart phone or a RFID tag, watch, shoe or bracelet. The mechanism of tracking would render useless if the device is not with patient when wandering episode occurs. The concept relies on user's memory and ability of independent living, which may not be dependable in case of dementia. Device maintenance is another issue that might prove to be cumbersome. Some design suggestions regarding mobile device includes easy integration in daily routine [59], portable size and weight [61], [59], [60], and disguised or less visible [61], [60]. In the outdoor scenario, it is evident that cellular phone or smart phone is frequently employed. Although pervasive and programmable, in the user study conducted by Robinson et al. [59], elderly participants mentioned their discomfort in using mobile phone. It needs to be charged and switched on routinely, which could be difficult to maintain for a dementia patient on their own, rendering the device unreliable. The user interface is comparatively complicated and might be unfamiliar as the phone may accommodate a range of applications along with the applications aimed to aid the dementia patients. On the other hand, mobile phones are preferable for their usability in making and receiving voice calls [61].

2) Alert Button and Safe Zones: Tradeoff between privacy and safety, a discussion that came up in user studies, is balanced in some systems using safe-zone alarms, where location data in transmitted to trusted caregiver only when patient venture outside of predetermined boundary, as opposed to track and store data continuously. Incidentally, the concepts of safe-zones and alert notifications [61], [59], [60] have also been discussed in need-finding studies. Several systems we mentioned integrate these ideas in their algorithms. A distinguishable, easily accessible, dedicated alert button on the device may be used in notifying caregiver or emergency services of emergency situations through signal or message [61], [60].

3) User Interface: A simple user interface is desirable to ensure ease of use in emergency situations. Systems running their applications on smart phones with other software, in case of application malfunction or shut down, would be challenging if a manual restart is required. In contrast, at present, smart phones are pervasive, carried everywhere and less conspicuous; an additional device attached to a person may draw unwanted attention. Moreover, technologies to aid other dementia symptoms, if necessary, may have to be integrated into the same device or system ecology, increasing complexity. Dedicated buttons for issuing alerts are proposed by users, which is difficult to achieve in multi-purpose devices. The user studies reveal a need for simple user interface that is easy to use for patient with dementia [61]. Various exclusive buttons have been suggested to increase ease of use - 'Call Home' button to call primary caregiver number [61], 'Alert' or 'I need help' button to announce emergency [61], [60], 'Route' button to request navigation [61]. An 'Alert' button on the caregiver device is also suggested, that would trigger tracking of the patient's device.

\section{4) Navigation, Communication and Remote Monitoring:}

To increase independence in travel, navigational tools, with similar navigational interface as familiar pre-existing devices [61] [60], are suggested in user study, for guiding a user to a destination [59] or assist in finding route to a known place. Several such system proposals were found during our survey. Global Positioning System(GPS) is the most prominent technology utilized to localize an individual, experiments, and evaluation proving it to be quite effective and adequately reliable in this domain. It is feasible in terms of availability, portability, and expense. In the user study [60], it is mentioned that the internet is not preferred as communication medium for lack of usage skills of dementia patients, whereas mobile network-based communication (phone calls and text messages) are preferred. In practice, the internet is the most popular medium utilized for data transmission, followed by mobile network communication modules. Two-way communication between caregiver and patient is suggested in [61], [59], [60]. Remote monitoring of a patient may reduce caregiver burden and stress as well as to ensure patient safety. It would be a useful feature if the monitoring system has provisions to handle purposeful change of route and breaks without alerting caregivers. To maintain patient privacy, the tracking could be triggered to start, by pressing a button or from lack of response from patient. All time online tracking is also suggested [61]. In fact, in practice, both all-time tracking and selected tracking have been considered by the proposed frameworks. Furthermore, tracking information should be sent to caregiver chosen by the patient [59].

\section{B. Special Features}

In addition to a general system design, some special features are proposed in the literature to enhance user comfort and reduce false positive warnings. Distance between caregivers and person with dementia could be measured to establish if patient is accompanied by a known person [40]. Speed of travel can be utilized to automatically detect travel mode (motor vehicle or walking) [40], [8] and define separate intervention and 
notification protocols for different scenarios. Even if patient is inside a marked safe zone, he or she might be wandering due to spatial disorientation. A panic button feature may enable her to trigger an alert situation [40]. In contrast, if person with dementia is travelling outside the safe zone on purpose, an option to enable daily mode or travel mode may also help reduce unnecessary warnings. Wandering at an unsafe hour within a safe zone can be managed by time surveillance [40]. Patient location data could be sent to caregivers periodically at scheduled intervals or only when an abnormal situation occurs. A special feature proposed by [41] enables caregiver to receive last ten locations of person with dementia immediately upon pressing a panic button. System failure warnings such as low battery alarm and inactivity alarm [41] are effective in avoiding further mishaps.

\section{Challenges in Experiment Design}

Experiments conducted for researches regarding algorithm formulation require user participation for data collection. Due to ethical constraints surrounding experiments involving human subjects, data are not publicly available. We notice that most experiments are conducted on data sets that are quite limited in size collected from a limited number of subjects. Therefore, it is possible that the results suffer from experimental setup biases and do not necessarily reflect real world scenarios. Moreover, comparison among different approaches and algorithms are not feasible as data, platform, environment, experiment design is quite different. For example, some algorithms are based on room to room movement where each room is treated as discrete point in trajectory [16], [17], whereas other algorithms consider consecutive co-ordinate in space, as discrete points in trajectory [6], [46]. Moreover, all algorithms are based on generic models of the aforementioned patterns. In reality, the patterns may vary depending on the person, differentiation between wandering and purposeful movements may not be so straightforward. In short, methodologies in devising and evaluating the algorithms differ in terms of utilized technologies, collected data, experiment ecology and study subjects. Moreover, we particularly notice redefinition of or deviation from the basic patterns to fit them in environmental setting or scenarios.

\section{Merging Indoor and Outdoor Scenarios}

A single system, that considers various scenarios (i.e. both indoor and outdoor) or definitions of wandering would be complex to design and implement. GPS (Global Positioning System) is a prevalent technology in research endeavors regarding tracking patients in outdoor environment [6], [10] [11], [12], [13], [62]. On the other hand, detecting wandering in an indoor environment needs a different set of equipment, that is suitable for a smaller area of travel - RFID tags, magnetometers as sensory devices and local computer as remote server for data storage and calculation. Some solutions accommodate sending notifications to other end users, to seek for help or informing current status. Some create interventions for person with dementia to coerce them to a predefined, beneficial action. It is apparent that in the indoor setting, RFID sensor data (Cartesian space coordinates) are prevalent, whereas GPS data (Longitude, Latitude) is used in the outdoor experiments. A challenge is to seamlessly merge the two scenarios under one algorithm or system; for example, upon detection of home-zone, the indoor wandering detection sub-component would be turned on. The problem arises when person with dementia ventures into an unknown indoor environment. There, technology like RFID, with wall mounted sensors of limited range, will not be feasible. Again, GPS data is not precise enough to detect patterns in a confined, smaller area.

\section{E. Effect of Emerging Technologies}

Most, if not all, systems that we mention here, leverage technologies, frameworks or algorithms from computing and electronics domain, rather than being built exclusively for the medical domain. As a result, technology, used in building a system, change or rather evolve with new inventions in various sectors in information technology industry. This is clearly evident when we compare 'Opportunity Knocks', which was proposed in 2004, with similar solutions like 'iWander' [8], 'iRoute' [37] and 'LaCasa' [15], which were published in 2010, 2011 and 2012 respectively. To collect data, Opportunity Knocks uses Bluetooth sensor beacon and General Packet Radio Service (GPRS) enabled cell-phone. The sensor beacon sends information to cell-phone, which in turn forwards this information to a remote server, that computes the location of the user using Geographic Information System (GIS) database. Merging these various platforms robustly and efficiently is challenging, considering connectivity, latency and data loss. In subsequent solutions [37], [8], [15], researchers moved on to Android smart phones, which have comparatively advanced location sensory and storage mechanisms (GPS, Google map and Cloud database), leveraging applications embedded in the same device. Functionality of the social network application (the study was done in 2009) developed in [33] mirrors social media applications of the present time. Rather than building a new framework, integrating this application to a more prevalent, robust, secure social media platform would be more efficient and relevant in the present context.

\section{F. Challenges in Practical Use}

Any technology employed, should be feasible to use in real life, especially for dementia patients. Here, the trade-off is between increasing privacy of the patient and reducing risks. It is crucial to maintain connectivity at most times and to ensure that the sensors are triggered by targeted behaviors without fail. Additionally, contingency plans should be in place to account for the failure of the primary technology. Patient needs to carry the equipment at all times for optimal result. Ensuring comfort and physical safety is of paramount importance. In human centered research, an important consideration is to select a technology that does not hinder the safety and comfort of the patients. Drawing examples from the discussed systems, the application proposed in [8] accommodates several promising aspects. This can be integrated with Android devices (i.e. mobile cell phones), used regularly, thus eliminating the need to carry additional technology. Most features are automated and do not require a feedback from user, which is convenient in this domain. Learning capability of detection component makes the system customizable for an individual. One drawback of the system is that the patient must carry the device while travelling outside. If lost or forgotten, system might provide faulty information or gather incorrect training data. It utilizes 
several application layer services (Google map, Google Voice call, audio prompt, Text messaging and Email applications) to produce alert messages. Therefore, it is required that the applications are available to be invoked when needed. It is claimed, that data collected over time improves the prediction performance of the system, and more usage results in improved accuracy. We notice the system proposed in [32] needs to act as an intelligent agent, without human intervention. This publication is from 2004; we notice, from later studies, that location map component of the system can be replaced by dynamic Google map. Two different devices and two modes of media are being used, to ensure message delivery to caregiver, which introduces another layer of connectivity. The authors also mention the dimension of the device $(51 \mathrm{~mm} \times 34 \mathrm{~mm} \times$ $16 \mathrm{~mm}$ and $27 \mathrm{~g}$ ), which seems feasible to carry around, which is an important aspect in terms of user comfort. There is always a trade-off between usability and performance of devices that are required to maintain online connectivity. The data communication process via a third party, may introduce considerable latency. Also, this procedure requires all-time connectivity, data transmission and power supply of equipment. Several features mentioned in the user studies, are implemented in commercial devices also, for example alert buttons, safe-zones and longer battery life.

\section{CONCLUSion}

With the ever-growing rate of dementia patients, it is imperative to have automated technological systems to increase independence in daily living and reduce accidents and stress. Wandering, being a pervasive behavior in persons with dementia, may result in unpredictable, insecure situations. We set out to address this problem, assembled literature to understand wandering behavior and how technology can assist in managing this behavior. Our survey revealed systems can be classified according to the perception of wandering, environmental setting, and underlying algorithms. Researchers integrate existing sensor, communication, hardware, and software technology to model a solution suitable for wandering behavior identification, as well as the issuance of notification and intervention. Several studies attempt to formulate algorithms to identify patterns in movement trajectories. While some components of proposed systems are parallel to results from need-finding user studies, there are areas where human computer interaction-based research would help in developing user and domain centering features.

\section{REFERENCES}

[1] M. Prince, A. Comas-Herrera, M. Knapp, M. Guerchet, and M. Karagiannidou, "World alzheimer report 2016: improving healthcare for people living with dementia: coverage, quality and costs now and in the future," 2016.

[2] A. Association et al., "2017 alzheimer's disease facts and figures," Alzheimer's \& Dementia, vol. 13, no. 4, pp. 325-373, 2017.

[3] R. G. Logsdon, L. Teri, S. M. McCurry, L. E. Gibbons, W. A. Kukull, and E. B. Larson, "Wandering: a significant problem among community residing individuals with alzheimer's disease," The Journals of Gerontology Series B: Psychological Sciences and Social Sciences, vol. 53, no. 5, pp. P294-P299, 1998.

[4] D. L. Algase, D. H. Moore, C. Vandeweerd, and D. Gavin-Dreschnack, "Mapping the maze of terms and definitions in dementia-related wandering," Aging \& mental health, vol. 11, no. 6, pp. 686-698, 2007.
[5] J. Corey-Bloom and D. Galasko, "Adjunctive therapy in patients with alzheimer's disease," Drugs \& aging, vol. 7, no. 2, pp. 79-87, 1995.

[6] Q. Lin, D. Zhang, X. Huang, H. Ni, and X. Zhou, "Detecting wandering behavior based on gps traces for elders with dementia," in Control Automation Robotics \& Vision (ICARCV), 2012 12th International Conference on. IEEE, 2012, pp. 672-677.

[7] B. Reisberg, S. H. Ferris, M. J. de Leon, and T. Crook, "The global deterioration scale for assessment of primary degenerative dementia." The American journal of psychiatry, 1982.

[8] F. Sposaro, J. Danielson, and G. Tyson, "iwander: An android application for dementia patients," in Engineering in Medicine and Biology Society $(E M B C), 2010$ annual international conference of the IEEE. IEEE, 2010, pp. 3875-3878.

[9] F. Miskelly, "Electronic tracking of patients with dementia and wandering using mobile phone technology," Age and ageing, vol. 34, no. 5, pp. 497-498, 2005.

[10] A. Solanas, E. Batista, F. Borras, A. Martínez-Ballesté, and C. Patsakis, "Wandering analysis with mobile phones: On the relation between randomness and wandering," in Pervasive and Embedded Computing and Communication Systems (PECCS), 2015 International Conference on. IEEE, 2015, pp. 168-173.

[11] Q. Lin, D. Zhang, K. Connelly, H. Ni, Z. Yu, and X. Zhou, "Disorientation detection by mining gps trajectories for cognitively-impaired elders," Pervasive and Mobile Computing, vol. 19, pp. 71-85, 2015.

[12] E. Batista, F. Borras, F. Casino, and A. Solanas, "A study on the detection of wandering patterns in human trajectories," in Information, Intelligence, Systems and Applications (IISA), 2015 6th International Conference on. IEEE, 2015, pp. 1-6.

[13] Y.-J. Chang, "Anomaly detection for travelling individuals with cognitive impairments," ACM SIGACCESS Accessibility and Computing, no. 97, pp. 25-32, 2010.

[14] D. J. Patterson, L. Liao, K. Gajos, M. Collier, N. Livic, K. Olson, S. Wang, D. Fox, and H. Kautz, "Opportunity knocks: A system to provide cognitive assistance with transportation services," in International Conference on Ubiquitous Computing. Springer, 2004, pp. 433-450.

[15] J. Hoey, X. Yang, E. Quintana, and J. Favela, "Lacasa: Location and context-aware safety assistant," in Pervasive Computing Technologies for Healthcare (PervasiveHealth), 2012 6th International Conference on. IEEE, 2012, pp. 171-174.

[16] N. Vuong, S. Chan, C. Lau, and K. Lau, "Feasibility study of a real-time wandering detection algorithm for dementia patients," in Proceedings of the First ACM MobiHoc Workshop on Pervasive Wireless Healthcare. ACM, 2011, p. 11.

[17] N. K. Vuong, S. Chan, and C. T. Lau, "Automated detection of wandering patterns in people with dementia," Gerontechnology, vol. 12, no. 3, pp. 127-147, 2014.

[18] W. Kearns, V. Nams, J. Fozard et al., "Tortuosity in movement paths is related to cognitive impairment," Methods Inf Med, vol. 49, no. 6, pp. 592-598, 2010.

[19] K. Makimoto, M. Yamakawa, N. Ashida, Y. Kang, and K.-R. Shin, "Japan-korea joint project on monitoring people with dementia," in 11th World Congress on the Internet and Medicine, 2006.

[20] C. Greiner, K. Makimoto, M. Suzuki, M. Yamakawa, and N. Ashida, "Feasibility study of the integrated circuit tag monitoring system for dementia residents in japan," American Journal of Alzheimer's Disease \& Other Dementias $($, vol. 22, no. 2, pp. 129-136, 2007.

[21] D. Martino-Saltzman, B. B. Blasch, R. D. Morris, and L. W. McNeal, "Travel behavior of nursing home residents perceived as wanderers and nonwanderers," The Gerontologist, vol. 31, no. 5, pp. 666-672, 1991.

[22] K. Ota, Y. Ota, M. Otsu, and A. Kajiwara, "Elderly-care motion sensor using uwb-ir," in Sensors Applications Symposium (SAS), 2011 IEEE. IEEE, 2011, pp. 159-162.

[23] M. Rowe, S. Lane, and C. Phipps, "Carewatch: a home monitoring system for use in homes of persons with cognitive impairment," Topics in geriatric rehabilitation, vol. 23, no. 1, p. 3, 2007.

[24] K. Doughty, G. Williams, P. King, and R. Woods, "Diana-a telecare system for supporting dementia sufferers in the community," in Engineering in Medicine and Biology Society, 1998. Proceedings of the 20th Annual International Conference of the IEEE, vol. 4. IEEE, 1998, pp. 1980-1983. 
[25] Y. Masuda, T. Yoshimura, K. Nakajima, M. Nambu, T. Hayakawa, and T. Tamura, "Unconstrained monitoring of prevention of wandering the elderly," in Engineering in Medicine and Biology, 2002. 24th Annual Conference and the Annual Fall Meeting of the Biomedical Engineering Society EMBS/BMES Conference, 2002. Proceedings of the Second Joint, vol. 3. IEEE, 2002, pp. 1906-1907.

[26] C. Nugent and J. Augusto, "A system for activity monitoring and patient tracking in a smart hospital," Smart Homes and Beyond: ICOST, p. 196, 2006.

[27] W. D. Kearns, J. L. Fozard, V. O. Nams, and J. D. Craighead, "Wireless telesurveillance system for detecting dementia," Gerontechnology, p. 90, 2011.

[28] T. Toutountzi, S. Phan, and F. Makedon, "A framework for the assessment of wandering behavior," in Proceedings of the 8th ACM International Conference on PErvasive Technologies Related to Assistive Environments. ACM, 2015, p. 93.

[29] D. L. Algase, E. R. Beattie, E.-L. Bogue, and L. Yao, "The algase wandering scale: initial psychometrics of a new caregiver reporting

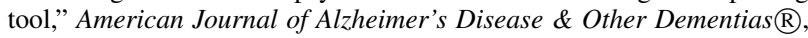
vol. 16 , no. 3, pp. 141-152, 2001.

[30] N. Vuong, S. Chan, C. Lau, and K. Lau, "A predictive location-aware algorithm for dementia care," in Consumer Electronics (ISCE), 2011 IEEE 15th International Symposium on. IEEE, 2011, pp. 339-342.

[31] K. Shimizu, K. Kawamura, and K. Yamamoto, "Location system for dementia wandering," in Engineering in Medicine and Biology Society, 2000. Proceedings of the 22nd Annual International Conference of the IEEE, vol. 2. IEEE, 2000, pp. 1556-1559.

[32] H. Ogawa, Y. Yonezawa, H. Maki, H. Sato, and W. M. Caldwell, "A mobile phone-based safety support system for wandering elderly persons," in Engineering in Medicine and Biology Society, 2004. IEMBS'04. 26th Annual International Conference of the IEEE, vol. 2. IEEE, 2004, pp. 3316-3317.

[33] R. Calvo-Palomino, P. De Las Heras-quiros, J. A. Santos-Cadenas, R. Román-López, and D. Izquierdo-Cortázar, "Outdoors monitoring of elderly people assisted by compass, gps and mobile social network," in International Work-Conference on Artificial Neural Networks. Springer, 2009, pp. 808-811.

[34] J. Wan, C. Byrne, G. M. O'Hare, and M. J. O'Grady, "Outcare: Supporting dementia patients in outdoor scenarios," in International Conference on Knowledge-Based and Intelligent Information and Engineering Systems. Springer, 2010, pp. 365-374.

[35] _ _ "Orange alerts: Lessons from an outdoor case study," in Pervasive Computing Technologies for Healthcare (PervasiveHealth), 2011 5th International Conference on. IEEE, 2011, pp. 446-451.

[36] S. Matsuoka, H. Ogawa, H. Maki, Y. Yonezawa, and W. M. Caldwell, "A new safety support system for wandering elderly persons," in Engineering in Medicine and Biology Society, EMBC, 2011 Annual International Conference of the IEEE. IEEE, 2011, pp. 5232-5235.

[37] S. Hossain, K. Hallenborg, and Y. Demazeau, "iroute: Cognitive support for independent living using bdi agent deliberation," in Trends in Practical Applications of Agents and Multiagent Systems. Springer, 2011, pp. 41-50.

[38] Y. K. Yuce and K. H. Gulkesen, "Development of a social support intervention with a network of caregivers to find wandering alzheimer's patients as soon as possible: A social computing application in healthcare," in Health Informatics and Bioinformatics (HIBIT), 2013 8th International Symposium on. IEEE, 2013, pp. 1-8.

[39] C.-Y. Ko, F.-Y. Leu, and I.-T. Lin, "A wandering path tracking and fall detection system for people with dementia," in Broadband and Wireless Computing, Communication and Applications (BWCCA), 2014 Ninth International Conference on. IEEE, 2014, pp. 306-311.

[40] P. A. Moreno, M. E. Hernando, and E. J. Gómez, "Design and technical evaluation of an enhanced location-awareness service enabler for spatial disorientation management of elderly with mild cognitive impairment," IEEE journal of biomedical and health informatics, vol. 19, no. 1, pp. 37-43, 2015.

[41] E. Batista, F. Borràs, and A. Martínez-Ballesté, "Monitoring people with mci: Deployment in a real scenario for low-budget smartphones,' in Information, Intelligence, Systems and Applications (IISA), 2015 6th International Conference on. IEEE, 2015, pp. 1-6.

[42] J. Ng and H. Kong, "Not all who wander are lost: Smart tracker for people with dementia," in Proceedings of the 2016 CHI Conference Extended Abstracts on Human Factors in Computing Systems. ACM, 2016, pp. 2241-2248.

[43] M. Mulvenna, S. Martin, S. Sävenstedt, J. Bengtsson, F. Meiland, R. M. Dröes, M. Hettinga, F. Moelaert, and D. Craig, "Designing \& evaluating a cognitive prosthetic for people with mild dementia," in Proceedings of the 28th Annual European Conference on Cognitive Ergonomics. ACM, 2010, pp. 11-18.

[44] C. C. Robusto, "The cosine-haversine formula," The American Mathematical Monthly, vol. 64, no. 1, pp. 38-40, 1957.

[45] N. Vuong, S. Chan, C. Lau, S. Chan, P. L. K. Yap, and A. Chen, "Preliminary results of using inertial sensors to detect dementia-related wandering patterns," in Engineering in Medicine and Biology Society (EMBC), 2015 37th Annual International Conference of the IEEE. IEEE, 2015, pp. 3703-3706.

[46] A. Kumar, C. T. Lau, S. Chan, M. Ma, and W. D. Kearns, "A unified grid-based wandering pattern detection algorithm," in Engineering in Medicine and Biology Society (EMBC), 2016 IEEE 38th Annual International Conference of the. IEEE, 2016, pp. 5401-5404.

[47] J. Petzold, F. Bagci, W. Trumler, and T. Ungerer, "Comparison of different methods for next location prediction," in European Conference on Parallel Processing. Springer, 2006, pp. 909-918.

[48] E. Estrada and J. A. Rodriguez-Velazquez, "Subgraph centrality in complex networks," Physical Review E, vol. 71, no. 5, p. 056103, 2005.

[49] T. S. Azevedo, R. L. Bezerra, C. A. Campos, and L. F. de Moraes, "An analysis of human mobility using real traces," in Wireless Communications and Networking Conference, 2009. WCNC 2009. IEEE. IEEE, 2009, pp. 1-6.

[50] 10 lifesaving location devices for dementia patients. Accessed: 2018-06-04. [Online]. Available: https://www.alzheimers.net/8-8-14location-devices-dementia/

[51] Trax. Accessed: 2018-06-04. [Online]. Available: https://traxfamily.com/

[52] Safe link. Accessed: 2018-06-04. [Online]. Available: http://safelinkgps.com/

[53] Pocketfinder. Accessed: 2018-06-04. [Online]. Available: http://pocketfinder.com/

[54] Mindme locate. Accessed: 2018-06-04. [Online]. Available: http://www.mindme.care/mindme-locate.html

[55] Smart sole. Accessed: 2018-06-04. [Online]. Available: http://gpssmartsole.com/gpssmartsole/

[56] itraq. Accessed: 2018-06-04. [Online]. Available: https://www.itraq.com/

[57] Angelsense. Accessed: 2018-06-04. [Online]. Available: https://www.angelsense.com/protect/dementia/

[58] Pli-1000 personal locator system. Accessed: 2018-06-04. [Online]. Available: https://projectlifesaver.org/locating-technology/pli1000-personal-locator-system/

[59] L. Robinson, K. Brittain, S. Lindsay, D. Jackson, and P. Olivier, "Keeping in touch everyday (kite) project: developing assistive technologies with people with dementia and their carers to promote independence," International Psychogeriatrics, vol. 21, no. 3, pp. 494-502, 2009.

[60] L. McCabe and A. Innes, "Supporting safe walking for people with dementia: User participation in the development of new technology," Gerontechnology, vol. 12, no. 1, pp. 4-15, 2013.

[61] K. Holbø, S. Bøthun, and Y. Dahl, "Safe walking technology for people with dementia: what do they want?" in Proceedings of the 15th International ACM SIGACCESS Conference on Computers and Accessibility. ACM, 2013, p. 21.

[62] L. Wan, C. Müller, V. Wulf, and D. W. Randall, "Addressing the subtleties in dementia care: pre-study \& evaluation of a gps monitoring system," in Proceedings of the SIGCHI Conference on Human Factors in Computing Systems. ACM, 2014, pp. 3987-3996. 